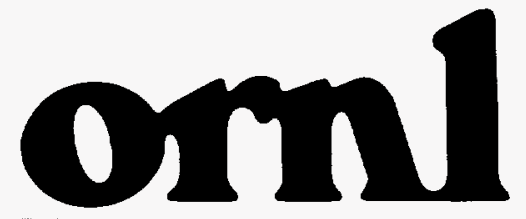

1)

OAK RIDGE

NATIONAL

LABORATORY
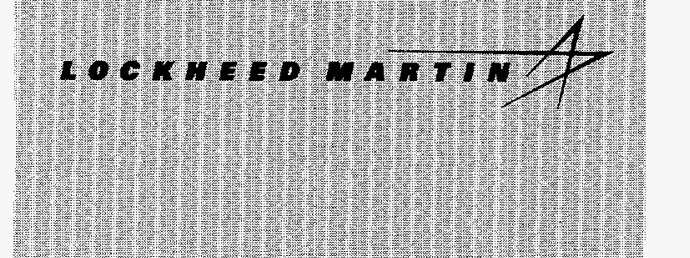

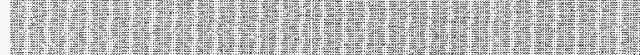

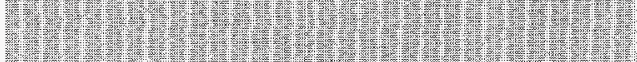

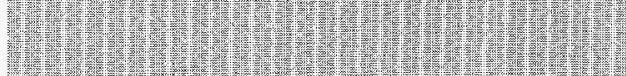

M

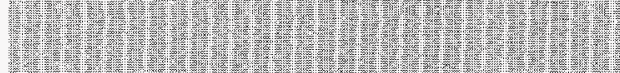

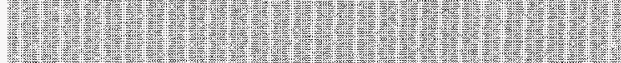

3)
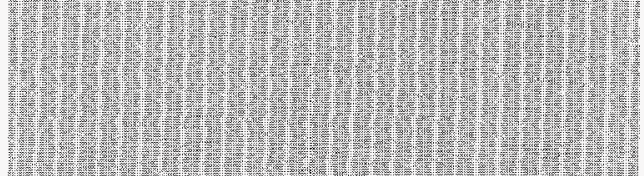

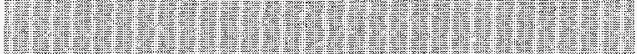
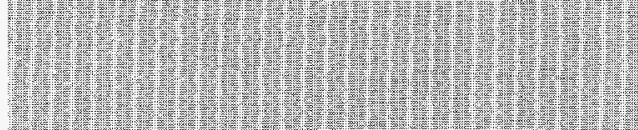

औrit:

$+$

MANAGED AND OPERATED BY

LOCKHEED WARTIN ENERGY RESEARCH CORPORATION FOR THE UMTED STATES

DEPARTUENT OF ENERGY

$$
\begin{aligned}
& \text { RECEIVEO } \\
& \text { SEP } 1 \text { ? } 1998 \\
& \text { OSTI }
\end{aligned}
$$

\section{On the Parallelization of the Acoustic Wave Equation with Absorbing Boundary Conditions}

\author{
C. T. White \\ V. A. Protopopescu \\ J. Barhen
}

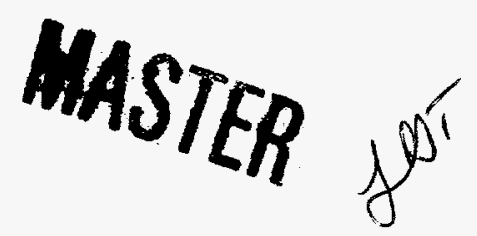

DIOTPABUTLN OF THIS DOCUMET is UNKMTEDE 
This report has been reproduced directly from the best available copy.

Available to DOE and DOE contractors from the Office of Scientific and Technical Information, P.O. Box $6 \dot{2}$, Oak Ridge, TN 37831; prices available from (615) 576-8401.

Available to the public from the National Technical Information Service, U.S. Department of Comrnerce, 5285 Port Royal Rd., Springfield, VA 22161.

This report was prepared as an account of work sponsored by an agency of the United States Crovernment. Neither the United States nor any agency thereof, nor any of their employees, makes any warranty, express or implied, or assumes any legal. liability or responsibility for the accuracy, completeness, or usefulness of any information, apparatus, product, or process disclosed, or represents that its use would not infringe privately owned rights. Reference herein to any specific commercial product, process, or service by trade name, trademark, manufacturer, or otherwise, does not necessarily constitute or imply its endorsement, recommendation, or favoring by the United States Government or any agency thereof. The views and opinions of authors expressed herein do not necessarily state or reflect those of the United States Government or any a.gency thereof. 


\section{DISCLAIMER}

Portions of this document may be illegible in electronic image products. Images are produced from the best available original document. 


\title{
ON THE PARALLELIZATION OF THE ACOUSTIC WAVE EQUA WITH ABSORBING BOUNDARY CONDITIONS
}

C. T. White

Department of Mathematics

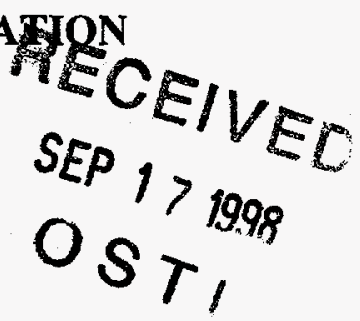

California Institute of Technology

1200 East California Boulevard

Pasadena, CA 91125

E-mail: cwhite@cco.caltech.edu

\author{
V. A. Protopopescu \\ J. Barhen \\ Center for Engineering Systems Advanced Research (CESAR) \\ Oak Ridge National Laboratory, P. O. Box 2008 \\ Oak Ridge, TN 37831-6355 \\ E-mail:vvp@ornl.gov,barhenj@ornl.gov
}

July 1998

Research supported by the Office of Fossil Energy, U. S. Department of Energy under the Advanced Computing Technology Initiative.

Prepared by the

Oak Ridge National Laboratory

Oak Ridge, Tennessee 37831

managed by

Lockheed Martin Energy Research Corp.

for the

U. S. DEPARTMENT OF ENERGY

Under Contract No. DE-AC05-96OR22464 
Contents

ABSTRACT

1 INTRODUCTION 1

2 BACKGROUND 2

2.1 ABSORBING BOUNDARY CONDITIONS FOR THE ACOUSTIC WAVE EQUATION . . . . . . . . . . . . . . . 2

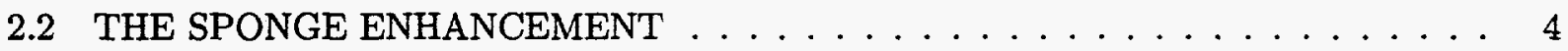

2.3 THE IFP-3D CODE $\ldots \ldots \ldots \ldots \ldots \ldots \ldots \ldots \ldots$

3 DISCRETE ABSORBING BOUNDARY CONDITIONS $\quad 7$

4 PARAllelization OF ACOUSTIC WAVE EQUATION 9

4.1 EXACT DIAGONALIZATION OF THE ACOUSTIC WAVE EQUATION WITH DIRICHLET BOUNDARY CONDITIONS $\ldots \ldots \ldots \ldots \ldots$

4.2 IMPOSSIBILITY OF EXACT DIAGONALIZATION OF THE ACOUSTIC WAVE EQUATION WITH ABSORBING BOUNDARY CONDITIONS $\ldots \ldots \ldots \ldots$

5 DISCUSSION 14

$\begin{array}{ll}\text { ACKNOWLEDGEMENTS } & 17\end{array}$

$\begin{array}{lr}\text { REFERENCES } & 19\end{array}$ 


\section{ABSTRACT}

Many practical problems involve wave propagation through atmosphere, oceans, or terrestrial crust. Modeling and analysis of these problems is usually done in (semi)infinite domains, but numerical calculations obviously impose restriction to finite domains. To mimic the actual behavior in the (semi)infinite medium, artificial absorbing boundary conditions are imposed at the boundaries, whereby waves can only exit, but not enter the finite computational domain. Efficient absorbing boundary conditions are difficult to analyze and costly to run. In particular, it is of interest to assess whether the wave equation with (approximate or exact) absorbing boundary conditions admits a suitable diagonalization. This would open the possibility for parallelizing many important numerical codes used in applications. In this paper we propose a set of stable, local, absorbing boundary conditions for the discrete acoustic wave equation. We show that the acoustic wave equation with absorbing boundary conditions cannot be exactly diagonalized.

\section{INTRODUCTION}

Propagation of acoustic, elastic, or electromagnetic waves in oceans, atmosphere, or terrestrial crust is often cast in the form of semi-infinite or infinite problems. Typical instances occur in underwater acoustics, seismic wave propagation, and electromagnetic wave generation by cosmic objects or antennas. However, for computational purposes these practically infinite problems must be recast within finite domains.

Several approaches have been investigated to address this requirement. One possibility is to map the (semi) infinite domain into a finite one. Another approach is to use smaller computational domains by substituting the true equations with simpler ones in the exterior domain. Then one can use the form of the solution of the simplified equations to construct boundary conditions for the true equations in the inner domain.

The most widely used approach has been to introduce artificial computational boundaries while maintaining the governing differential equations unchanged. On the artificial boundaries one should design boundary conditions that ensure that the incident waves are fully transmitted and the reflection is, ideally, zero. These artificial boundary conditions imposed on the solution of the wave equation in a (semi)infinite domain are called absorbing boundary conditions ( $\mathrm{ABCs}$ ). Various $\mathrm{ABCs}$ have been used in the numerical modeling of wave propagation (see for instance, [1-15] and references therein). Theoretically, pseudodifferential operators can be constructed that ensure perfect transmission (i.e., no reflection) of the outgoing waves at the artificial boundaries. Unfortunately, except in the one-dimensional case, these boundary conditions are non-local in both space and time and therefore not practical for numerical calculations. Resorting to local approximations is somewhat of a gamble since it may lead to ill-posed problems for the wave equation $[1,3,7]$. Even when the local approximations lead to well-posed problems, the perfectly absorbing property of the boundary is limited to certain angles of incidence (usually normal incidence) [1,7,9-12]. To enhance the (imperfect) absorptive quality of the artificial boundaries, additional damping and filtering mechanisms have been devised [4,5,14-16]. However, although they reduce reflection - sometimes to very low levels - dampers and sponges (or filters) introduce an extra computational burden and may inherently prevent the implementation of more efficient methods, such as parallelization. Even in the absence of sponges one faces an additional problem. Assuming that local stable approximations of $\mathrm{ABCs}$ have been constructed, these continuous boundary conditions have to be 
again approximated by discrete $\mathrm{ABCs}$ which are then coupled to a standard interior discretization of the wave equation. Since in this process some of the required properties may be lost, it seems more natural to look directly for local discrete versions of $A B C s$ that lead to stable, efficient, and possibly parallelizable schemes.

The aim of this paper is to analyze the parallelizability of the acoustic wave equation (AWE) with ABCs. The paper is structured as follows: In Section 2 we review the AWE and various ABCs and absorption enhancing techniques. We present a new local, stable, discrete $A B C$ for the exact discrete AWE in Section 3 and compare its performance with the three dimensional code developed by the Insitut Français du Petrcle (IFP-3D) [5]. Section 4 is devoted to an analysis of the ABCs as to their potential for parallelization. The results are analyzed in the final section.

\section{BACKGROUND}

\subsection{ABSORBING BOUNDARY CONDITIONS FOR THE ACOUSTIC WAVE EQUATION}

Let $u(x, t)$ solve the AWE

$$
u_{t t}-c^{2} \nabla \cdot \nabla u=g(x, t)
$$

in the domain $\Omega$. Usually this equation is supplemented with initial conditions, $u(x, t=0)=u_{0}(x)$, $u_{t}(x, t=0)=u_{1}(x)$ and with boundary conditions at the boundary of $\Omega, \partial \Omega$. The typical boundary conditions for the AWE are Dirichlet, Neumann, and Robin. The homogeneous Dirichlet condition is imposed at a fixed boundary and is expressed by

$$
\left.u\right|_{\partial \Omega}=0
$$

Waves striking a fixed boundary will reflect with an amplitude of opposite sign as the incoming wave. The homogeneous Neumarin condition is imposed at a free boundary and is expressed by

$$
\left.\frac{\partial u}{\partial n}\right|_{\partial \Omega}=0
$$

where $\boldsymbol{n}$ is the unit vector normal to the boundary surface. Waves reflect from such a boundary with the same amplitude as the incoming waves. The Robin condition is given by

$$
\frac{\partial u}{\partial n}+\left.\lambda u\right|_{\partial \Omega}=0
$$

where again, $\boldsymbol{n}$ is the unit vector normal to the boundary surface. This condition is also known as the mixed or elastic boundary condition and in order to obtain stable solutions, $\lambda$ is required to be greater than zero.

These boundary conditions, are cibviously non-absorbing. To effectively mimic an infinite physical domain within a finite computational domain we need artifical boundaries which almost completely "transmit" waves out of the region of computation. An exact solution to this problem exists only for 
the one-dimensional (1D) AWE. In higher dimensions, various approaches have been proposed to deal with this problem, often based on implementing approximations to pseudodifferential operators on the boundaries. We give a brief summary of the techniques used in Ref. 5:

The problem domain, $\Omega$, is assumed parallelepipedic. As such, conditions for the faces, edges, and corners are required. The homogeneous three dimensional (3D) AWE reads

$$
\left[\frac{1}{c^{2}} \frac{\partial^{2}}{\partial t^{2}}-\left(\frac{\partial^{2}}{\partial x^{2}}+\frac{\partial^{2}}{\partial y^{2}}+\frac{\partial^{2}}{\partial z^{2}}\right)\right] u=0 .
$$

To derive a boundary condition for the faces $x=X_{\min }$ or $x=X_{\max }$, we solve formally for $\frac{\partial u}{\partial x}$ :

$$
\frac{\partial u}{\partial x}= \pm\left(\frac{1}{c} \frac{\partial}{\partial t} \sqrt{1-c^{2}\left(\frac{\partial^{2}}{\partial t^{2}}\right)^{-1}\left(\frac{\partial^{2}}{\partial y^{2}}+\frac{\partial^{2}}{\partial z^{2}}\right)}\right) u
$$

For the face $x=X_{\min }$, we select the positive root; for the face $x=X_{\max }$, we choose the negative root. We show the details for the face $x=X_{\min }$.

The pseudodifferential operator in the RHS of Eq. (6) is approximated by using the Taylor expansion for the square root, $\sqrt{1-\alpha}=1-\frac{1}{2} \alpha+0\left(\alpha^{2}\right)$, yielding

$$
\frac{\partial^{2} u}{\partial x \partial t}=\left[\frac{1}{c} \frac{\partial^{2}}{\partial t^{2}}-\frac{1}{2} c\left(\frac{\partial^{2}}{\partial y^{2}}+\frac{\partial^{2}}{\partial z^{2}}\right)\right] u .
$$

For the face $x=X_{\min }$, we obtain

$$
\frac{1}{c^{2}} \frac{\partial^{2} u}{\partial t^{2}}+\frac{1}{c} \frac{\partial^{2} u}{\partial x \partial t}-\frac{1}{2}\left(\frac{\partial^{2} u}{\partial y^{2}}+\frac{\partial^{2} u}{\partial z^{2}}\right)=0 \text { on } x=X_{\min }
$$

This equation is called second order $\left(15^{\circ}\right)$ paraxial approximation of the acoustic waves equation in the direction $x>0[5]$.

For the surface $x=X_{\max }$, the corresponding ABC reads:

$$
\frac{1}{c^{2}} \frac{\partial^{2} u}{\partial t^{2}}-\frac{1}{c} \frac{\partial^{2} u}{\partial x \partial t}-\frac{1}{2}\left(\frac{\partial^{2} u}{\partial y^{2}}+\frac{\partial^{2} u}{\partial z^{2}}\right)=0 \text { on } x=X_{\max }
$$

The ABCs at the other surfaces have similar expressions, obtained by circular permutation of the coordinates.

Let us now consider the line defined by the intersection of the two surfaces $x=X_{\min }$ and $y=Y_{\min }$. The associated well-posed condition along this line is obtained by satisfying the ABCs used along each surface and the AWE.

The condition for the surfaces $x=X_{\min }$ and $y=Y_{\min }$ are

$$
\frac{1}{c^{2}} \frac{\partial^{2} u}{\partial t^{2}}-\frac{1}{c} \frac{\partial^{2} u}{\partial x \partial t}-\frac{1}{2}\left(\frac{\partial^{2} u}{\partial y^{2}}+\frac{\partial^{2} u}{\partial z^{2}}\right)=0
$$




$$
\frac{1}{c^{2}} \frac{\partial^{2} u}{\partial t^{2}}-\frac{1}{c} \frac{\partial^{2} u}{\partial y \partial t}-\frac{1}{2}\left(\frac{\partial^{2} u}{\partial x^{2}}+\frac{\partial^{2} u}{\partial z^{2}}\right)=0
$$

respectively.

Solving Eq. (8a) for $\frac{\partial^{2} u}{\partial y^{2}}$ and Eq. (8b) for $\frac{\partial^{2} u}{\partial z^{2}}$, and substituting into the AWE, Eq. (5), we obtain

$$
\frac{3}{2} \frac{1}{c^{2}} \frac{\partial^{2} u}{\partial t^{2}}+\frac{1}{c} \frac{\partial}{\partial t}\left(\frac{\partial u}{\partial x}+\frac{\partial u}{\partial y}\right)-\frac{1}{2} \frac{\partial^{2} u}{\partial x^{2}}=0
$$

Similarly, we deduce the conditicns for the others edges.

Finally, let us consider the four corners at the bottom of the model. For example, the $A B C$ at the corner defined by the intersectior. of the surfaces $x=X_{\min }, y=Y_{\min }$, and $z=Z_{\min }$, is obtained by the combination of the three ABCs along these surfaces and the AWE. The condition for the lines $\left\{x=X_{\min }, y=Y_{\min }\right\},\left\{x=X_{\min }, z=Z_{\min }\right\},\left\{y=Y_{\min }, z=Z_{\min }\right\}$ are

$$
\begin{aligned}
& \frac{3}{2} \frac{1}{c^{2}} \frac{\partial^{2} u}{\partial t^{2}}-\frac{1}{c} \frac{\partial}{\partial t}\left(\frac{\partial u}{\partial x}+\frac{\partial u}{\partial y}\right)-\frac{1}{2} \frac{\partial^{2} u}{\partial z^{2}}=0 \\
& \frac{3}{2} \frac{1}{c^{2}} \frac{\partial^{2} u}{\partial t^{2}}-\frac{1}{c} \frac{\partial}{\partial t}\left(\frac{\partial u}{\partial x}+\frac{\partial u}{\partial z}\right)-\frac{1}{2} \frac{\partial^{2} u}{\partial y^{2}}=0 \\
& \frac{3}{2} \frac{1}{c^{2}} \frac{\partial^{2} u}{\partial t^{2}}-\frac{1}{c} \frac{\partial}{\partial t}\left(\frac{\partial u}{\partial y}+\frac{\partial u}{\partial z}\right)-\frac{1}{2} \frac{\partial^{2} u}{\partial x^{2}}=0
\end{aligned}
$$

respectively. Solving Eq. (10a) for $\frac{\partial^{2} u}{\partial z^{2}}$, Eq. (10b) for $\frac{\partial^{2} u}{\partial y^{2}}$, and Eq. (10c) for $\frac{\partial^{2} u}{\partial x^{2}}$, and substituting these expressions into the AWE, Eq. (5), we obtain the $\mathrm{ABC}$ at the corner $\left\{x=X_{\min }, y=\right.$ $\left.Y_{\min }, z=Z_{\min }\right\}$

$$
\frac{2}{c^{2}} \frac{\partial^{2} u}{\partial t^{2}}-\frac{1}{c} \frac{\partial}{\partial t}\left(\frac{\partial u}{\partial x}+\frac{\partial u}{\partial y}+\frac{\partial u}{\partial z}\right)=0 .
$$

No additional conditions are needed for the four corners of the free surface.

\subsection{THE SPONGE ENHANCEMENT}

An alternative approach to reducing reflection from the boundaries is based on sponge filters that are located near the boundary of the domain. Their intended effect is to damp waves traveling from the boundary into the interior (thereby reducing the amplitude of reflected waves), and leave unaffected the waves approachirig the boundary. Of course, the sponge filter may be used in conjunction with some form of $A B C$ s for enhanced overall effect. For illustration, we derive the sponge filter for the $1 \mathrm{D}$ situation. The $1 \mathrm{D}$ AWE can be written as

$$
\left(\frac{1}{c} \frac{\partial}{\partial t}-\frac{\partial}{\partial x}\right)\left(\frac{1}{c} \frac{\partial}{\partial t}+\frac{\partial}{\partial x}\right) u=f
$$


Each of these first order operators in the L.H.S. of Eq. (12) governs the propagation of waves in a single direction: waves traveling right satisfy $\frac{1}{c} \frac{\partial u}{\partial t}-\frac{\partial u}{\partial x}=0$, while waves traveling left satisfy $\frac{1}{c} \frac{\partial u}{\partial t}+\frac{\partial u}{\partial x}=0$.

Near the right boundary we introduce an artificial source that propagates waves to the left with a negative amplitude, thus partially or - in the ideal situation - completely canceling the waves reflected from the boundary. We express this as follows:

$$
\frac{1}{c^{2}} \frac{\partial^{2} u}{\partial t^{2}}-\frac{\partial^{2} u}{\partial x^{2}}=f-\epsilon(x)\left(\frac{1}{c} \frac{\partial u}{\partial t}+\frac{\partial u}{\partial x}\right)
$$

The term $\epsilon(x)$ allows us to control the damping factor at each spatial point, so that we may take it to be zero deep in the interior of the domain and increasing as we approach the right boundary. We note that large changes in $\epsilon$ over a short distance tend to introduce additional reflections. Thus, for the sponge term to be effective, the damping coefficient must increase gradually, often over a large number of grid points.

In $3 \mathrm{D}$ the situation is more complicated since the factorization above does not apply. The goal, however, remains to damp waves traveling in from the boundaries. For plane geometry, large surfaces and homogeneous media, the $1 \mathrm{D}$ approximation of the sponge at the $x=X_{\max }$ boundary,

$$
\frac{1}{c^{2}} \frac{\partial^{2} u}{\partial t^{2}}-\left(\frac{\partial^{2} u}{\partial x^{2}}+\frac{\partial^{2} u}{\partial y^{2}}+\frac{\partial^{2} u}{\partial z^{2}}\right)=f-\epsilon(x)\left(\frac{1}{c} \frac{\partial u}{\partial t}+\frac{\partial u}{\partial x}\right)
$$

works reasonably well. Similar terms are used for the other faces.

An enhancement to the "one-way" sponge filter described above is obtained by damping both incoming and outgoing waves near the boundary [4]. While providing a greater net damping effect, (as waves are damped both before and after they reflect from a boundary), this approach may require one to disregard data obtained from points within the sponge filter, since even outgoing waves have been disturbed at these points.

We construct the "two-way" sponge by simply introducing, as sources, canceling factors for both incoming and outgoing waves. For the 3D AWE near the $x=X_{\max }$ boundary, we find

$$
\begin{aligned}
\frac{1}{c^{2}} \frac{\partial^{2} u}{\partial t^{2}}-\left(\frac{\partial^{2} u}{\partial x^{2}}+\frac{\partial^{2} u}{\partial y^{2}}+\frac{\partial^{2} u}{\partial z^{2}}\right) & =f-\epsilon(x)\left(\frac{1}{c} \frac{\partial u}{\partial t}+\frac{\partial u}{\partial x}\right)-\epsilon(x)\left(\frac{1}{c} \frac{\partial u}{\partial t}-\frac{\partial u}{\partial x}\right) \\
& =f-\frac{2}{c} \epsilon(x) \frac{\partial u}{\partial t}
\end{aligned}
$$

The damping term $\frac{\partial u}{\partial t}$ involves now a coefficient that depends on the speed and on the distance to the boundary. Similar terms describe "two-way" sponges for the other faces.

\subsection{THE IFP-3D CODE}

We include here some brief comments on the three dimensional code developed by the Insitut Français du Petrole (IFP-3D) to solve the AWE for use in geophysical exploration and analysis 
[5]. This code is designed for seismic studies of subsurface exploration. This feature alone requires massive calculations to account for each point in the discrete 3D domain. Additional features of the code also play a part in its total computational complexity which results in a rather costly program.

The main features of the IFP-3D code are:

- full 3D model of the AWE in a parallelepipedic domain;

- implements the second order paraxial boundary conditions on five of the six faces of the domain, implements either the Dirichlet or Neumann condition on the sixth face to simulate either a fixed or free surface;

- includes an additional one-way sponge enhancement to further reduce reflections;

- assumes a uniform spatial grid, i.e. $\Delta_{x}=\Delta_{y}=\Delta_{z}$;

- uses an explicit scheme: a finite difference scheme approximates time derivatives to second order and space derivatives to tenth order (where possible);

- is sequential in time: solves updates for all spatial points at a given time step before proceeding to the next;

- allows the use of several source terms, including a point source given by a Ricker function; and

- writes output for specific lines or planes of points in the domain which are the "simulated receivers."

The most consequential drawbacls of the IFP code are:

- the explicit method is only conditionally stable and requires rather small $\Delta_{t}$ (hence a large number of time steps) while allowing only relatively low source frequencies;

- the algorithm is essentially sequential in time: current attempts at parallelizing the IFP-3D code involve stepping sequentially through time, with costly node communications required at each step; and

- there is a high computational cost associated with the code's approximation of ABCs: in addition to the often mentioned cost involved in the paraxial boundary conditions, there is also the high cost of perforrning the sponge update on several grid layers (often 20 or more) near the boundary.

Our research has been motivated in large part by the need to enhance the efficiency of the 3D model algorithms (in particular the IFP-3D code) while not sacrificing their accuracy. This requires the development of (hopefully unconditionally) stable algorithms that have potential to support parallel implementations. The following analysis focuses on these issues. 


\section{DISCRETE ABSORBING BOUNDARY CONDITIONS}

In this section we derive discrete $\mathrm{ABCs}$ which are local in both time and space and considerably simpler in expression than the paraxial equations used in Ref. 5. As mentioned before, our motivation in constructing these boundary conditions comes from not only the computational complexity of the paraxial equations, but also from the difficulty encountered when trying to incorporate the paraxial equations into a time parallel algorithm. Moreover, we have chosen to start directly with discrete $\mathrm{ABCs}$ since, eventually, the numerical solution of the problem requires discretization. We first give a brief account of the derivation of these conditions, followed by an analysis of their effectiveness.

In constructing the discrete $\mathrm{ABCs}$, we assume that the 3D (parallelipipedic) domain is discretized in the form:

$$
(0,1, \ldots, N X+1) \times(0,1, \ldots, N Y+1) \times(0,1, \ldots, N Z+1)
$$

The boundaries, in the form of surfaces, edges, or corners have at least one coordinate equal to either $0, N X+1, N Y+1$, or $N Z+1$.

The new ABCs generalize to the multidimensional case the method of characteristics that works exactly in the $1 D$ problem. We will now show details of the new boundary conditions for the $3 D$ problem; a similar procedure applies in the $2 \mathrm{D}$ case.

We denote by $u^{[n]}(i, j, k)$ the solution of the discrete 3D AWE at discrete time $n$ and grid point $(i, j, k)$. The discrete homogeneous 3D AWE obtained from the finite difference method with second order approximations reads:

$$
\begin{aligned}
u^{[n+1]}(i, j, k)-2 u^{[n]}(i, j, k)+u^{[n-1]}(i, j, k)= & p(i, j, k)\left(u^{[n]}(i+1, j, k)+u^{[n]}(i-1, j, k)\right. \\
& +u^{[n]}(i, j+1, k)+u^{[n]}(i, j-1, k) \\
& \left.+u^{[n]}(i, j, k+1)+u^{[n]}(i, j, k-1)-6 u^{[n]}(i, j, k)\right)
\end{aligned}
$$

where $p(i, j, k)=\frac{c^{2}(i, j, k) \Delta_{t}^{2}}{h^{2}}, c(i, j, k)$ is the speed of sound at the grid point $(i, j, k), \Delta_{t}$ is the discrete time step, and $h=\Delta_{x}=\Delta_{y}=\Delta_{z}$ is the discrete spatial step.

We write $u^{[u+1]}$ and $u^{[n-1]}$ by using the ansatz

$$
\begin{aligned}
u^{[n+1]}(i, j, k)= & p(i, j, k)\left[u^{[n]}(i+1, j, k)+\frac{1}{2}\left(u^{[n]}(i, j+1, k)+u^{[n]}(i, j-1, k)\right.\right. \\
& \left.\left.+u^{[n]}(i, j, k+1)+u^{[n]}(i, j, k-1)\right)\right]+(1-3 p(i, j, k)) u^{[n]}(i, j, k) \\
u^{[n-1]}(i, j, k)= & p(i, j, k)\left[u^{[n]}(i-1, j, k)+\frac{1}{2}\left(u^{[n]}(i, j+1, k)+u^{[n]}(i, j-1, k)\right.\right. \\
& \left.\left.+u^{[n]}(i, j, k+1)+u^{[n]}(i, j, k-1)\right)\right]+(1-3 p(i, j, k)) u^{[n]}(i, j, k) .
\end{aligned}
$$


which clearly satisfy Eq. (16).

For $i=0,1 \leq j \leq N Y, 1 \leq k \leq N Z$, Eq. (17a) becomes

$$
\begin{aligned}
u^{[n+1]}(0, j, k)= & p(0, j, k)\left[u^{[n]}(1, j, k)+\frac{1}{2}\left(u^{[n]}(0, j+1, k)+u^{[n]}(0, j-1, k)\right.\right. \\
& \left.\left.+u^{[n]}(0, j, k+1)+u^{[n]}(0, j, k-1)\right)\right]+(1-3 p(0, j, k)) u^{[n]}(0, j, k)
\end{aligned}
$$

For each face we perform a similar splitting, which selects only the interior point in the direction normal to the surface. To derive the edge condtions, we split the two spatial second derivates normal to the intersecting faces, solving the equation which contains only those interior points. For example, at $i=0, j=0,1 \leq k \leq N Z$, we obtain

$$
\begin{aligned}
u^{[n+1]}(0,0, k)= & p(0,0, k)\left[u^{[n]}(1,0, k)+u^{[n]}(0,1, k)\right. \\
& \left.+\frac{1}{2}\left(u^{[n]}(0,0, k+1)+u^{[n]}(0,0, k-1)\right)\right]+(1-3 p(0,0, k)) u^{[n]}(0,0, k) .
\end{aligned}
$$

Finally, for corner points we split all three of the spatial derivates, again solving the subequation which contains only these intericr points. For the corner $i=0, j=0, k=0$, we get

$$
\begin{aligned}
u^{[n+1]}(0,0,0)= & p(0,0,0)\left[u^{[n]}(1,0,0)+u^{[n]}(0,1,0)+u^{[n]}(0,0,1)\right] \\
& +(1-3 p(0,0,0)) u^{[n]}(0,0,0) .
\end{aligned}
$$

These new $\mathrm{ABCs}$ are.now local in both time and space and are compatible with the discretized equation used for the interior of the domain.

To analyze the effectiveness of these $A B C s$ we compared them with the second order paraxial conditions used in the IFP-3D algorithm and with the Dirichlet condition. We used two discrete domains with $150 \times 150 \times 100$ and $150 \times 150 \times 80$ grid points, respectively. The source is located $(75,75,75)$ in the center of the domain in the $X Y$ plane, 25 (or 5) points from the bottom face boundary. A single receiver is placed initially at the same point as the source; on later shots it is moved along the $Z=75$ plane to realize various angles of incident waves namely $0^{\circ}, 17^{\circ}, 25^{\circ}$, and $41^{\circ}$. The speed of sound, $c$, is equal to $750.00 \mathrm{~m} / \mathrm{s}, \Delta_{t}=.167 \times 10^{-2} \mathrm{~s}, \Delta_{x}=\Delta_{y}=\Delta_{z}=3.0 \mathrm{~m}$, and the central frequency of the Richer source is $20.00 \mathrm{~Hz}$.

In evaluating the effectiveness of the new $\mathrm{ABCs}$, we considered the stated objectives: simplicity, speed, and potential for parallelization. Specifically, one must decide if the absorption of the new ABCs reaches an acceptable level, and if the greater absorption provided by the paraxial conditions is not offset by their complexity. In particular, for the serial implementation we are interested in the time necessary to calculate the boundary update at each time step. Also, since the complex paraxial ABCs cannot be easily incorporated into a fully parallel scheme, one must assess whether the simpler $\mathrm{ABCs}$ will allow the simultaneous diagonalization of the resulting system, providing full space and time parallelism.

Figures 1 and 2 show the relative reduction in amplitudes of reflected waves for both the paraxial and new ABCs. One sees a greater reduction from the paraxial conditions, but both significantly 
reduce the unabsorbed Dirichlet reflection. Since the tests were performed on relatively small scale models, we have no definitive conclusions regarding the computation times of the various boundary conditions. Preliminary data show that the new ABCs are calculated slightly faster than the paraxial ABCs. Due to locality, this effect would be significantly enhanced by parallelization.

\section{PARALLELIZATION OF ACOUSTIC WAVE EQUATION}

The implementation of time parallelism for numerical solutions of partial differential equations has been shown to hold great potential [19-21]. Whenever applicable, this formalism would enable the use of fully implicit methods which, due to their unconditional stability, dramatically reduce the number of required time steps and provide a massive degree of coarse-grain temporal parallelism with minimal communication and synchronization requirements. Such algorithms have proven to be highly suitable for implementation on massively parallel MIMD architectures such as the Cray T3D and the Intel Paragon [19-21 and references therein]. We shall assess the applicability of this methodology to the AWE with $\mathrm{ABCs}$, with specific implementation targeted at the enhancement of the IFP-3D code. The approach we follow is to search for the eigenvalue-eigenvector decomposition of the matrices resulting from the finite difference method. To illustrate this procedure we use the AWE in a finite uniform domain with Dirichlet boundary conditions and then turn to the situation involving $\mathrm{ABCs}$.

\subsection{EXACT DIAGONALIZATION OF THE ACOUSTIC WAVE EQUATION WITH DIRICHLET BOUNDARY CONDITIONS}

Once again, assume that the parallelipedic domain is discretized in the form

$$
(0,1, \ldots, N X+1) \times(0,1, \ldots, N Y+1) \times(0,1, \ldots, N Z+1)
$$

Recall that the boundaries are comprised of those points having at least one coordinate equal to 0 , $N X+1, N Y+1$, or $N Z+1$.

We consider the discrete system obtained through the finite difference method,

$$
\mathbf{u}^{[n+1]}-2 \mathbf{u}^{[n]}+\mathbf{u}^{[n-1]}=p \mathcal{L} \mathbf{u}^{[n]}+p \mathbf{f}^{[n]}
$$

where

1. $\mathbf{u}^{[n]}$ is an $(N X) \times(N Y) \times(N Z)$ - vector (note that we may omit boundary points from the vector $\mathbf{u}$ since we are assuming Dirichlet (zero) boundary conditions);

2. $\mathcal{L}$ is the block tridiagonal $[(N X) \times(N Y) \times(N Z)]^{2}$ discrete Laplacian matrix [19];

3. $p=\frac{c^{2} \Delta_{t}^{2}}{h^{2}}$ is constant throughout the domain, and $h=\Delta_{x}=\Delta_{y}=\Delta_{z} ;$ and

4. $\mathrm{f}^{[n]}$ is the source function at time $t=n \Delta_{t}$. 


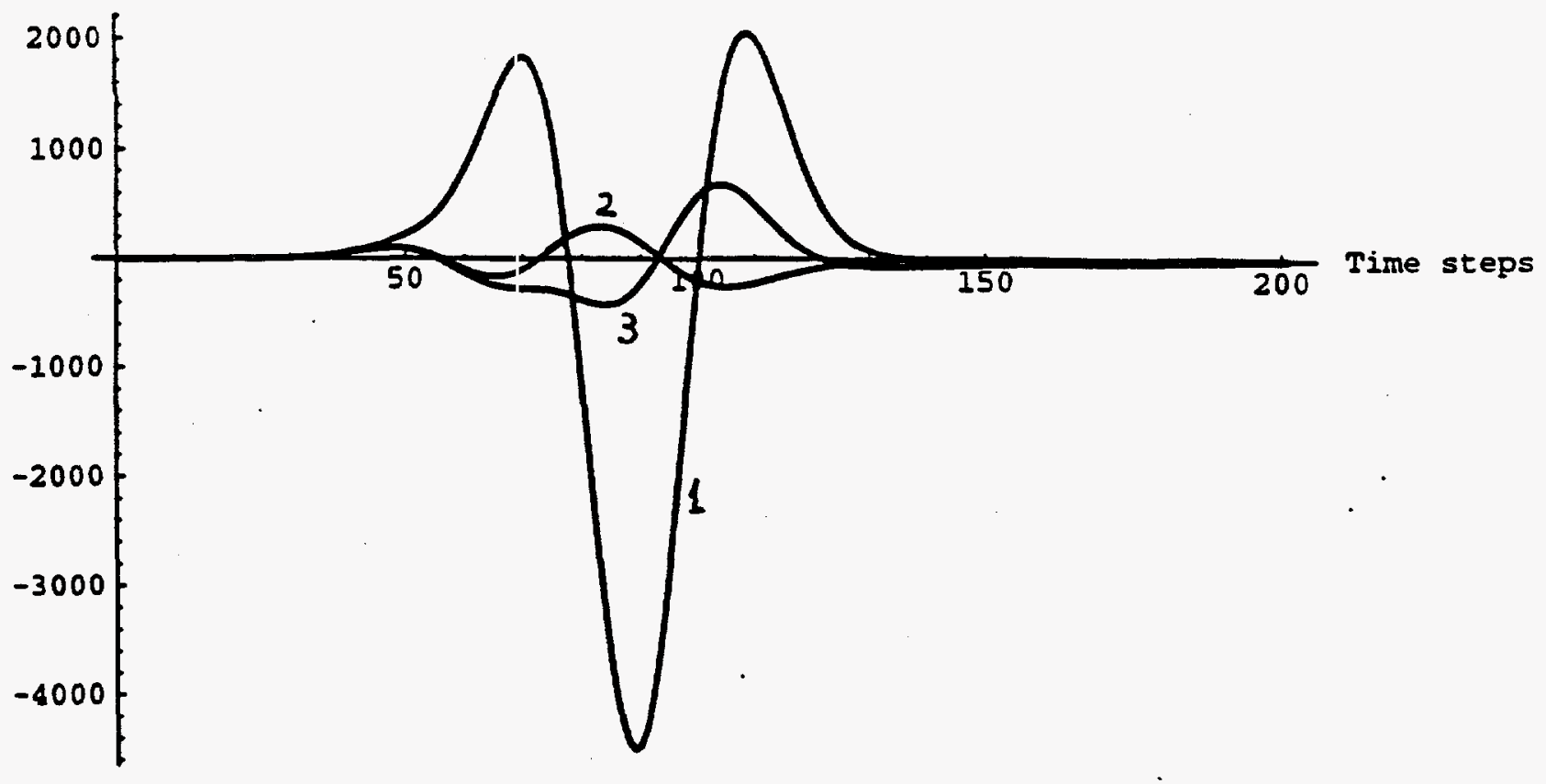

Fig. 1. Amplitude of reflected waves as a function of time for the $150 \times 150 \times 80$ domain at $0^{\circ}$ : Dirichlet (1), IFP-3D (2), new ABCs (3).

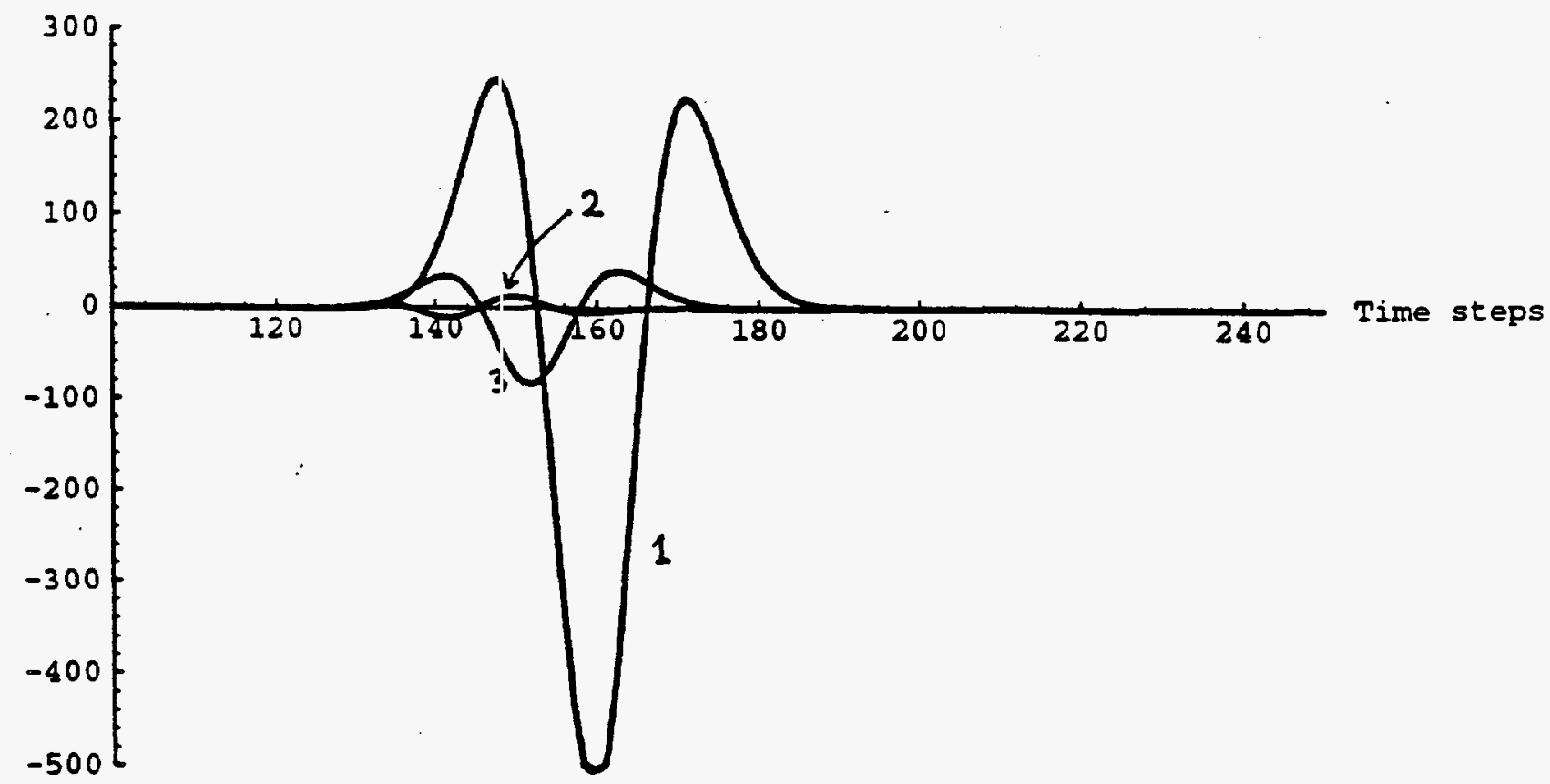

Fig. 2. Amplitude of reflected waves as a function of time for the $150 \times 150 \times 100$ domain at $0^{\circ}$ : Dirichlet (1), IFP-3D (2), new ABCs (3). 
The Crank-Nicholson approximation:

$$
\mathcal{L} \mathrm{u}^{[n]} \approx \frac{1}{2}\left(\mathcal{L} \mathrm{u}^{[n+1]}+\mathcal{L} \mathrm{u}^{[n-1]}\right)
$$

results in the system

$$
\left(I-\frac{1}{2} p \mathcal{L}\right) \mathbf{u}^{[n+1]}=2 \mathbf{u}^{[n]}-\left(I-\frac{1}{2} p \mathcal{L}\right) \mathbf{u}^{[n-1]}+p \mathrm{f}^{[n]}
$$

The eigenvalue-eigenvector decomposition of the discrete Laplacian $\mathcal{L}$ is known analytically [19]. We do mention that $\mathcal{L}$ is diagonalized by an orthogonal transformation matrix $\Theta$ resulting from a discrete sine transform. Let $\Theta \mathcal{L} \Theta^{-1}=\Lambda$ be diagonal, where of course $\Theta^{-1}=\Theta^{T}$.

Therefore,

$$
\Theta\left(I-\frac{1}{2} p \mathcal{L}\right) \Theta^{-1} \Theta \mathbf{u}^{[n+1]}=2 \Theta \mathbf{u}^{[n]}-\Theta\left(I-\frac{1}{2} p \mathcal{L}\right) \Theta \Theta^{-1} \mathbf{u}^{[n-1]}+p \Theta \mathrm{f}^{[n]}
$$

By making the substitution $\mathbf{v}^{[n]}=\Theta \mathbf{u}^{[n]}$, we obtain the decoupled system

$$
\left(I-\frac{1}{2} p \Lambda\right) \mathbf{v}^{[n+1]}=2 \mathbf{v}^{[n]}-\left(I-\frac{1}{2} p \Lambda\right) \mathbf{v}^{[n-1]}+\mathbf{g}^{[n]}
$$

where $\mathbf{g}^{[n]}=p \Theta \mathbf{f}^{[n]}$. Note that the eigenvalues of $\mathcal{L}$ are negative, so that $\left(I-\frac{1}{2} p \Lambda\right)$ is nonsingular. Let $D=\left(I-\frac{1}{2} p \Lambda\right)^{-1}$ and rewrite the above system as

$$
\mathbf{v}^{[n+1]}=2 D \mathbf{v}^{[n]}-\mathbf{v}^{[n-1]}+D \mathbf{g}^{[n]}
$$

If the above two-term recursion is homogenous, its iteration involves a linear combination (with coefficients which depend upon the initial conditions) of powers of the matrices $D \pm\left(D^{2}-I\right)^{1 / 2}$, which may be computed very efficiently since $D$ is diagonal. If, on the other hand, the above is a nonhomogeneous recursion, we may still efficiently apply standard algorithms such as recuvsive doubling or cyclic reduction, since $D$ is diagonal. All that remains is to use the stored matrix $\Theta^{-1}=\Theta^{T}$ to perform the transformation of $\mathbf{v}$ back to $\mathbf{u}$. Finally, note the unconditional stability of this method resulting from the Crank-Nicholson approach.

\subsection{IMPOSSIBILITY OF EXACT DIAGONALIZATION OF THE ACOUS- TIC WAVE EQUATION WITH ABSORBING BOUNDARY CONDITIONS}

The diagonalization approach presented in the previous section can be carried out for periodic and Neumann boundary condition, but cannot be immediately generalized to more complicated boundary conditions. Indeed, the difficulty in diagonalizing the AWE with the second order paraxial boundary conditions was among the motivating factors of our present analysis. We shall show that, the paraxial boundary conditions as well as our other proposed schemes fail to meet specific conditions necessary in order to diagonalize exactly the corresponding AWE problem either in contunious or discrete form. We now turn to an analysis of these conditions. 
First we consider an AWE which is continuous in time and discrete in space. In general, this can be written in the form:

$$
\mathcal{X} \frac{\partial^{2} \mathbf{u}}{\partial t^{2}}+\mathcal{Y} \frac{\partial \mathbf{u}}{\partial t}=\mathcal{Z} \mathbf{u}, \quad \text { for } \mathcal{X}, \mathcal{Y}, \mathcal{Z} \in M_{N}(\Re)
$$

where $M_{N}(\Re)$ denotes the set of the $N \times N$ matrices over the real numbers $\Re$.

We require that Eq. (21) with suitable $\mathrm{ABCs}$ satisfy the following three conditions:

I. closely approximates an undamped discrete AWE in the interior of the domain; this ensures that the $\mathrm{ABCs}$ do not distcrit the interior solution.

II. provides absorbing, or non-reflecting boundaries, i.e. approximates well a (semi)infinite medium; and

III. admits a full, simultaneous diagonalization of the three matrices involved which ensures the potential for parallelization. The simultaneous diagonalization requires the existence of two matrices $\Theta, \Psi \in M_{N}(\Re), \Psi$ invertible, such that $\Theta \mathcal{X} \Psi, \Theta \mathcal{Y} \Psi, \Theta \mathcal{Z} \Psi$ are all diagonal matrices.

Before establishing conditions neressary for a triple $(\mathcal{X}, \mathcal{Y}, \mathcal{Z})$ to meet these three criteria, we state a weaker version of II which will be useful later.

$\mathrm{II}^{\prime}$. for any initial condition, Eq.(1) and $g(x, t)=0$ has a unique solution, $\mathbf{u}(t)$, such that $\lim _{t \rightarrow \infty} \|$ $\mathbf{u}(t) \|=0$. This conditions ensures that after a finite time; the wave will have essentially left the finite medium (no reflections).

Suppose that II $^{\prime}$ and III hold for some $\mathcal{X}, \mathcal{Y}, \mathcal{Z} \in M_{N}(\Re)$. Let $\Theta, \Psi$ be the matrices from III above and define $\Theta \mathcal{X} \Psi=\Phi, \Theta \mathcal{Y} \Psi=\Gamma, \Theta \mathcal{Z} \Psi=\Lambda$.

Defining $\mathbf{v}(t)=\Psi^{-1} \mathbf{u}(t)$, we obtain the following decoupled system:

$$
\Phi \frac{\partial^{2} \mathbf{v}}{\partial t^{2}}+\Gamma \frac{\partial \mathbf{v}}{\partial t}=\Lambda \mathbf{v}
$$

The differential equation associatied to a typical mode is of the form

$$
\phi v^{\prime \prime}+\gamma v^{\prime}=\lambda v
$$

Since Eq. (21) is second order in time, Eq. (23) will also be specified with two initial data. Therefore, in order to ensure the existence ar.d uniqueness of solutions to Eq. (23), and hence Eq. (21), it follows that $\phi \neq 0$.

Indeed, if $\phi=\gamma=\lambda=0$, then any twice differentiable function $v(t)$ satisfying the initial conditions also satisfies the (trivial) DE. Thus, solutions are not unique. On the other hand, if $\phi=0$ while not both $\gamma$ and $\lambda$ are zero then the DE in Eq. (23) is of order one or zero. Since two initial data have been specified, this problem. is in general overdetermined and solutions need not exist.

From this observation, we conclude that both $\mathcal{X}$ and $\Theta$ are nonsingular. Without loss of generality, therefore, we may assume $\mathcal{X}=I$. 
We now consider solutions to Eq. $(23)$ where $\phi \neq 0$. Since

$$
\|\mathbf{v}(t)\|=\left\|\Psi^{-1} \mathbf{u}(t)\right\| \leq\left\|\Psi^{-1}\right\|\|\mathbf{u}(t)\|
$$

we obtain the series of implications

$$
\|\mathbf{u}(t)\| \rightarrow 0 \Longrightarrow\|\mathbf{v}(t)\| \rightarrow 0 \Longrightarrow v(t) \rightarrow 0 \quad \text { for each mode }
$$

This last implication enables us to conclude $\gamma \neq 0$ and $\lambda \neq 0$. Indeed, if $\gamma=\lambda=0$, then solutions to Eq. (23) are linear in time and in general do not decay. If $\gamma \neq 0, \lambda=0$, then solutions to Eq. (23) involve a constant plus an exponential; certain choices of initial conditions guarantee that this constant is non-zero. Finally, if $\gamma=0, \lambda \neq 0$, the roots of the characteristic equation of Eq. (23) are $\pm \sqrt{\lambda / \phi}$. If $\operatorname{Re}(\sqrt{\lambda / \phi}) \neq 0$, then one of the two exponentials will grow unboundedly with time. On the other hand, if $\operatorname{Re}(\sqrt{\lambda / \phi})=0$, then the solution will have a constant magnitude, which will be nonzero for some initial conditions.

Thus, in every case except $\gamma \neq 0$ and $\lambda \neq 0$, the mode in question fails to decay in time for certain initial conditions. Then, by the above remark, $\|\mathbf{u}(t)\|$ does not converge to zero. Thus, we must have $\gamma \neq 0$ and $\lambda \neq 0$; hence $\Gamma$ and $\Lambda$, (consequently $\mathcal{Y}$ and $\mathcal{Z}$ ), are non-singular.

We now argue that $\mathcal{Y}$ and $\mathcal{Z}$ commute, and thus we may take $\Psi=\Theta^{-1}$. Indeed, assuming the simultaneous diagonalization as before, we obtain

$$
\begin{aligned}
\mathcal{Y Z} & =\Theta^{-1} \Gamma \Psi^{-1} \Theta^{-1} \Lambda \Psi^{-1}=\Theta^{-1} \Gamma \Phi^{-1} \Lambda \Psi^{-1} \\
& =\Theta^{-1} \Lambda \Phi^{-1} \Gamma \Psi^{-1}=\Theta^{-1} \Lambda \Psi^{-1} \Theta^{-1} \Gamma \Psi^{-1}=\mathcal{Z Y}
\end{aligned}
$$

Finally, since any finite collection of pairwise commuting, diagonalizable matrices can be simultaneously diagonalized via a similarity transformation, we may take $\Psi=\Theta^{-1}$.

In summary:

If we suppose that the system

$$
\frac{\partial^{2} \mathbf{u}}{\partial t^{2}}+\mathcal{Y} \frac{\partial \mathbf{u}}{\partial t}=\mathcal{Z} \mathbf{u}
$$

is $(i)$ is simultaneously diagonalizable and $(i i)$ has solutions that vanish for large $t$, then the following necessarily hold:

1. $\mathcal{Y}$ and $\mathcal{Z}$ are non-singular;

2. $\mathcal{Y} \mathcal{Z}=\mathcal{Z} \mathcal{Y}$; and

3. The system can be simultaneously diagonalized by a similarity transformation.

As before, $\mathcal{Z}$ is the discrete Laplacian matrix for the interior points (plus some suitable expression for the boundary points), and hence it cannot be a diagonal matrix. Therefore, since $\mathcal{Y}$ commutes 
with $\mathcal{Z}$, if $\mathcal{Y}$ is a diagonal matrix then $\mathcal{Y}=\gamma I_{N}$ for some $\gamma \in \Re$. But this will introduce a constant absorption of the waves throughcut the whole medium and not only near the boundaries. Thus for $\gamma \neq 0$, condition I will not be satisfied.

We turn now to an AWE that is discretized not only in space but also in time and is represented by the second order recursion relation

$$
\mathcal{A} \mathbf{u}^{[n+1]}+\mathcal{B} \mathbf{u}^{[n]}+\mathcal{C} \mathbf{u}^{[n-1]}=0
$$

for $\mathcal{A}, \mathcal{B}, \mathcal{C} \in M_{N}(\Re)$.

As before, we suppose there exist $\Theta, \Psi \in M_{N}(\Re), \Psi$ invertible, such that $\hat{\mathcal{A}}=\Theta \mathcal{A} \Psi, \hat{\mathcal{B}}=\Theta \mathcal{B} \Psi$, $\hat{\mathcal{C}}=\Theta \mathcal{C} \Psi$ are all diagonal matrices. The resulting decoupled system is

$$
\hat{\mathcal{A}} \mathbf{v}^{[n+1]}+\hat{\mathcal{B}} \mathbf{v}^{[n]}+\hat{\mathcal{C}} \mathbf{v}^{[n-1]}=0
$$

where $\mathbf{v}^{[k]}=\Psi^{-1} \mathbf{u}^{[k]}$. Each mode is then a second order recursion of the form

$$
\alpha v^{[n+1]}+\beta v^{[n]}+\gamma v^{[n-1]}=0
$$

where $v_{0}$ and $v_{1}$ are specified initial conditions.

The analysis proceeds as in the continuous case. If $\alpha=\beta=\gamma=0$, then solutions to this trivial recursion relation are not unique. If either $\alpha=0, \beta \neq 0, \gamma \neq 0$ or $\gamma=0, \alpha \neq 0, \beta \neq 0$, then the above is actually a first order recurrence. The problem is overspecified since two initial terms are given, and in general, solutions need not exist. The same applies if two of the three coefficients are zero. Thus, we conclude that the matrices $\hat{\mathcal{A}}$ and $\hat{\mathcal{C}}$ are nonsingular. Therefore $\mathcal{A}, \mathcal{C}$, and $\Theta$ are also nonsingular.

Without loss of generality we can take $\mathcal{A}=I$. Furthermore, in order that $\Theta$ and $\Psi$ exist, it is necessary that $\mathcal{B}$ and $\mathcal{C}$ commute, hence $I, \mathcal{B}$, and $\mathcal{C}$ may be simultaneously diagonalized via a similarity transformation. Therefore, we may assume $\Psi=\Theta^{-1}$.

The general solutions of the recursion relation Eq. (27) with $\alpha=1$ is

$$
v^{[n]}=\lambda_{1} r_{1}^{n}+\lambda_{2} r_{2}^{n}
$$

where $r_{1}, r_{2}$ are the roots of the characteristic polynomial $x^{2}+\beta x+\gamma=0$, and $\lambda_{1}$ and $\lambda_{2}$ are complex constants determined by the initial conditions.

Thus, in order that $v^{[n]} \rightarrow 0$ as $n \rightarrow \infty$, irrespective of the initial conditions, a final necessary condition is

$$
0<\left|r_{1}\right|,\left|r_{2}\right|<1 \text {. }
$$

In summary, three conditions similar to (1) - (3) in the continuous case are shown to be necessary here as well. Also, as in the previous case, this will contradict at least one of the requirements I III. 


\section{DISCUSSION}

We conclude with a short discussion of these necessary conditions and, in particular, their impact on exact diagonalization of the AWE with ABCs. We shall first demonstrate how the ABCs discussed in this paper fail to meet at least one of the necessary conditions, thus establishing the impossibility of simultaneous diagonalization and parallelization for these ABCs.

The second order paraxial boundary conditions fail to meet the nonsingularity condition. Note, for example, that the corner condition involves only the first and second time derivatives of $u$ but not the function itself; therefore, in the notation of the preceding section, the matrix $\mathcal{Z}$ contains some rows comprised entirely of zeros and hence is singular.

The general sponge filter fails to meet the commutativity condition. Since a sponge filter involves only multiples of a time derivative at each point, the matrix $\mathcal{Y}$ (in the notation of the previous section) is diagonal. As it is that the matrix $\mathcal{Z}$ performs the discrete Laplacian updates on interior points, $\mathcal{Z}$ cannot itself be diagonal. Thus, $\mathcal{Y}$ and $\mathcal{Z}$ commute if and only if $\mathcal{Y}=\gamma I_{N}$ for some constant $\gamma$, and of course we are only interested in $\gamma>0$. Thus, the only sponges (used independently of other boundary routines) which satisfy the necessary conditions are those which have a constant damping factor. (Note that the constant factor throughout the domain is the coefficient of the time derivative, $\frac{c_{i j k}^{2} \Delta_{t}^{2}}{h^{2}} \epsilon_{i j k}$, and not simply $\epsilon$.)

Finally, the new $\mathrm{ABCs}$ fail to meet the nonsingularity condition as discussed in the previous section for the time-discretized system. Specifically, this condition requires that the matrices operating on the vectors $\mathbf{u}^{[n+1]}$ and $\mathbf{u}^{[n-1]}$ be nonsingular. Since the new ABCs make use of only the two time steps $(n+1) \Delta_{t}$ and $n \Delta_{t}$ in computing each update, the matrix operating on the vector $\mathbf{u}^{[n-1]}$ contains many rows consisting entirely of zeros and hence is singular.

As a means by which to introduce the general problem, consider first the special case of a uniform sponge, or, using an earlier notation, a constant diagonal matrix $\mathcal{Y}$. This method is an unacceptable solution to our problem, as it fails to restrict the region of damping to points close to the boundary, but instead damps waves uniformly throughout the domain. Thus, any damping effect one wishes to create on or near the boundary will also be created throughout the entire domain. Consequently, if the damping coefficient is large enough to reduce reflections to an acceptable level, it will similarly damp interior propagation and thereby destroy the accuracy of the interior solution.

The analysis of a general sponge (and matrix $\mathcal{Y}$ ) is an open problem that should be addressed after criteria I - II are given a more precise quantitative statement.

We also leave open at this point, the complete conclusions of our analysis of second order diagonalizable systems for solving the AWE. Whether or not there are any workable schemes which satisfy our necessary conditions is not known nor guessed at this point. One item to notice is that in the case of a diagonal $\mathcal{Y}$, the requirements that $\mathcal{Y}$ be nonsingular and commute with $\mathcal{Z}$ are somewhat competing notions, forcing the condition of uniformity which results in either insufficient damping at the boundary or excessive damping in the interior. We suggest that a similar incompatibility may exist for the more general situation. However, it is our belief that an analysis of this last question will rest upon a clear and quantitative reformulation of the requirement that any scheme respect the accuracy of the original AWE in the interior of the domain. We recommend this direction for future research. 
-

$\bullet$ 


\section{ACKNOWLEDGEMENTS}

This research was performed at the Center for Engineering Science Advanced research, Oak Ridge National Laboratory. Funding was provided by the Office of Fossil Energy under the Advanced Computing Technology Initiative under contract DE-AC05-960R22464 with Lockheed Martin Energy Research Corporation. The authors thank Drs. D. B. Semeraro and D. B. Reister for their help, and Dr. Leigh House (LANL) for continued support. 


\section{REFERENCES}

1. B. Engquist and A. Majda, Absorbing Boundary Conditions for the Numerical Simulations of Waves, Math. Comp, 31, 629 (1977).

2. B. Engquist and A. Majda, Radiation Boundary Conditions for Acoustic and Elastic Wave Calculations, Comm. Pure and Appl. Math, 32, 313 (1979).

3. M. Israeli and S.A. Orszag, Approximation of Radiation Boundary Conditions, J. Comp. Phys., 41, 115 (1981).

4. C. Cerjan, D. Kosloff, R. Kosloff, and M. Reshef, A Nonreflecting Boundary Condition for Discrete Boundary Condition for Discrete Acoustic and Elastic Wave Equations, Geophysics, 50, 705 (1985).

5. R.L. Higdon, Absorbing Boundary Conditions for Difference Approximations to the Multidimensional Wave Equation, Math. Comp., 47, 437 (1986).

6. R.L. Higdon, Numerical Absorbing Boundary Conditions for the Wave Equation, Math. Comp. 49, 65 (1987).

7. J. Sochacki, R. Kubichek, J. George, W.R. Fletcher, and S. Smithson, Absorbing Boundary Conditions and Surface Waves, Geophysics, 52, 60 (1987).

8. A. Bamberger, P. Joly, and Y.E. Roberts, Second Order Absorbing Boundary Conditions for the Wave Equations: A Solution for the Corner Problem, SIAM J. Numer. Anal., 27, 323 (1990).

9. L.T. Long and J.S. Liow, A Transparent Boundary for Finite Difference Wave Equation, Geophysics, 55, 201 (1990).

10. R.L. Higdon, Radiation Boundary Conditions for Elastic Wave Propagation, SIAM J. Numer. Anal., 27, 831 (1990).

11. D.R. Burns and R.A. Stephen, Three-Dimensional Numerical Modeling of Geoacoustical Scattering from Seafloor Topography, J. Acoust. Soc. Am., 88, 2338 (1990).

12. D. Givoli, Non-Reflecting Boundary Conditions, J. Comp. Phys. 94, 1 (1991).

13. L. Anné, J. Brac, and H. Tran, Propagation 3D d'Ondes Acoustiques, Institut Français du Pétrole, April 1993.

14. Chengbin Peng and M. Nafi Toksöz, An Optimal Absorbing Boundary Condition for Finite Difference Modeling of Acoustic and Elastic Wave Propagation, J. Acoust. Soc. Am., 95, 733 (1994).

15. J.P. Berenger, A Perfectly Matched Layer for the Absorption of Electromagnetic Waves, J. Comp. Phys., 114, 185 (1994).

16. J. Nordström, Accurate Solutions of the Navier-Stokes Equations Despite Unknown Outflow Boundary Data, J. Comp. Phys., 120, 184 (1995).

17. P. Joly and J. Tuomela, A New Theoretical Approach to Absorbing Layers, SIAM J. Numer. Anal., 34, 671 (1997). 
18. Xiaobing Feng, Absorbing Boundary Conditions for Electromagnetic Wave Propagation, Math. Comp., to appear.

19. N. Toomarian, A. Fijany, and J. Barhen, Time-Parallel Solution of Linear Partial Differential Equations on the Intel Touchstone Delta Supercomputer, Concurrency: Practice and Experience, 6, 641, (1994).

20. A. Fijany and P. C. Messina, Unconditionally Stable Explicit Method for Massively Parallel Solution of Acoustic Wave Equations, SPIE, 2571, 212 (1995)

21. A. Fijany, M. A. Jensen, Y. Rahmat-Samii, and J. Barhen, A Massively Parallel Computation Strategy for FDTD: Time and Space Parallelism Applied to Electromagnetics Problems, IEEE Trans. Ant. Prop. 43, 1441. (1995). 


\section{INTERNAL DISTRIBUTION}

1-5. J. Barhen

6. Y. Braiman

7. G. A. Geist

8. C. W. Glover

9. W. C. Grimmell

10. H. K. Liu

11. E. M. Oblow

12. L. E. Parker

13. N. Peterfreund

14 - 24. V. A. Protopopescu
25. N. S. Rao

26. D. B. Reister

27. M. S. Smith

28. C. Touzet

29. M. A. Unseren

30. S. Wiseman

31. T. Zacharia

32. Central Research Library

33. ORNL Laboratory Records - RC

34-35. ORNL Laboratory Records - OSTI

\section{EXTERNAL DISTRIBUTION}

36. Dr. Robert E. Price, ER-15, Office of Basic Energy Sciences, Department of Energy, Washington, DC 20585

37. Dr. Fred Aminzadeh, UNOCAL, 14141 SW Freeway, Suite 301-225, Sugarland TX 77478.

38. Dr. John Blair, JBX Technologies, 25 Moore Road, Wayland, MA 01778

39. Amir Fijany, Jet Propulsion Laboratory, California Institute of Technology, 4800 Oak Grove Drive., Pasadena, CA 91109

40. Dr. Oscar P. Manley, 13212 Wye Oak Drive, Gaithersburg, MD 20878.

41. Dr. Charles R. Weisbin, Robotics and Mars Exploration Technology Program, Jet 'Propulsion Laboratory, 4800 Oak Grove Drive; Pasadena, CA 91109

42 - 47. C. White, Mathematics Department, California Institute of Technology, 1200 East California Blvd. Pasadena, CA 91125 